\title{
BeppoSAX view of the NS-LMXB GS 1826-238
}

\author{
M. Cocchi ${ }^{1}$, R. Farinelli ${ }^{2,3}$, and A. Paizis ${ }^{4}$ \\ ${ }^{1}$ INAF, Istituto di Astrofisica Spaziale e Fisica Cosmica sez. di Roma, via Fosso del Cavaliere, 100, 00133 Roma, Italy \\ e-mail: Massimo.Cocchi@iasf-roma.inaf.it \\ 2 INAF, Istituto di Astrofisica Spaziale e Fisica Cosmica sez. di Palermo, Italy \\ e-mail: farinelli@ifc.inaf.it \\ 3 Dipartimento di Fisica, Universitá di Ferrara, Italy \\ ${ }^{4}$ INAF, Istituto di Astrofisica Spaziale e Fisica Cosmica sez. di Milano, Italy \\ e-mail: ada@iasf-mi.inaf.it
}

Received 1 December 2010 / Accepted 8 March 2011

\section{ABSTRACT}

\begin{abstract}
Context. The spectroscopic characteristics of GS 1826-238, a neutron star in a low-mass X-ray binary system, have already been studied by sensitive, wide band X-ray telescopes (e.g. BeppoSAX, RXTE, INTEGRAL). Up to now, the source has always been observed in a low-hard spectral state, with two spectral components typically detected. The persistent high-energy $(>10 \mathrm{keV})$ emission is effectively explained by thermal Comptonisation by a hot electron cloud $\left(k T_{\mathrm{e}} \sim 20 \mathrm{keV}\right)$; a further low energy component, modelled either by pure blackbody emission or by Compton-modified blackbody radiation by a few keV electron plasma, is generally needed to yield acceptable fits in the soft X-ray band.

Aims. The aim of the present work is to investigate the origin and the nature of the low energy emission of GS 1826-238 further, along with its contribution to the bolometric output of the source, dominated by the high-temperature thermally Comptonised radiation. Methods. This kind of investigation needs sensitive data in the widest available energy band. Simultaneous covering of both the soft X-rays (below $1 \mathrm{keV}$ ) and the hard X-rays (up to hundreds of $\mathrm{keV}$ ) is crucial for an unbiased characterisation of the two spectral components, so we searched the whole BeppoSAX-NFI archive for all the available GS 1826-238observations. We analysed a total of six data sets, collected from 1997 to 2000; data analysis of two of them was still unpublished. In this study we applied both a wellestablished (сомРтт) and a more recent, updated Comptonisation model (сомРтв), in order to get the widest quantitative information about the physical parameters at work

Results. Our results confirm that the $0.1-200 \mathrm{keV}$ emission of GS 1826-238 needs two components to be explained. In particular, two populations of soft seed photons, with different colour temperatures, are observed. One population is Comptonised to high energies by a hot electron cloud (temperatures in the range 19-24 keV, anticorrelated with the source luminosity), while the other is directly observed and can be modelled by a pure blackbody. We also propose an alternative model in which both the seed photon populations are Compton-modified by the electron plasma. This model explains the observed emission of GS 1826-238 as accurately as the traditional one and, moreover, fits well in a wider evolutionary scenario able to describe the state transitions observed in neutron-star low-mass X-ray binaries.

The use of сомРтв also indicates that, in the case of GS 1826-238, the seed photons populations are not distributed as a pure blackbody.
\end{abstract}

Key words. X-rays: general - X-rays: binaries - X-rays: individual: GS 1826-238 - radiation mechanisms: thermal

\section{Introduction}

GS $1826-238$ is a persistent low-mass X-ray binary (LMXB) harbouring a neutron star (NS). Discovered during a manoeuvre of the Ginga satellite (Makino et al. 1988) and previously regarded as a transient source, GS 1826-238 is actually a persistent emitter at least since the BeppoSAX era, and always observed in a low-hard spectral state (LHS) with its high-energy emission extending well above $100 \mathrm{keV}$.

Wide band LHS spectra of weakly magnetised NS are traditionally best described by two main components, each of them connected to a distinct soft photon population: 1) a Comptonmodified blackbody radiation with electron plasma temperatures in the range $\sim 15-30 \mathrm{keV}$, accounting for most of the source energy output and dominating in the hard X-rays; and 2) a directly observed blackbody (possibly a multicolour disk emission) with a different colour temperature with respect to the previous one that, though bolometrically weaker, becomes important in the soft X-rays characterising the spectral shape below $\sim 10 \mathrm{keV}$. In the LHS the blackbody seed photon population facing the inverse Compton scattering is usually hotter than the directly emerging one. It is supposedly located in the close vicinity of the NS (in the so-called boundary layer and/or in the photosphere of the NS itself), while the colder pseudo-blackbody population likely originates in the inner accretion disk.

This picture fits well into an eastern-like scenario (Mitsuda et al. 1989) for NS-LMXBs: in this traditional model the cold photon population is described by multicolour disk blackbody and is directly observed. The hotter population is (almost) completely Comptonised by a hot electron plasma. The opposite occurs in the (also traditional) western model (White et al. 1988): the cold, disk, population is Compton-upscattered by a radially wide electron corona, while the hotter, NS, population, modelled by pure blackbody, is directly detected. Both the models deal with two separate blackbody (or multicolour) distributed photon populations and a single Comptonising region. Here we term 
eastern-like a scenario where the NS population is Comptonised and western-like a scenario with Comptonised disk population, regardless of the energy distribution of the population themselves, which is likely non-ideal (multicolour) in its nature. Nowadays eastern-like models, having the advantage of keeping the X-ray emitting region very compact (hence naturally meeting the fast variability requirements set by the timing observations), are more popular, at least for NS-LMXBs in an LHS, where the coronal height scale is supposedly at its maximum. The previously reported spectral properties of GS 1826-238 make no exception.

Early, band-limited observations of GS 1826-238 were obtained by TTM (in 't Zand 1992), and ROSAT (Barret et al. 1995) at low energies, and by CGRO-OSSE (Strickman et al. 1996), in the hard X-rays, while the history of relatively recent, sensitive, wide band studies of GS 1826-238, which is in basic agremeent with the above two-component picture, mainly includes results from BeppoSAX (in 't Zand et al. 1999; Del Sordo et al. 1999), RXTE (Barret et al. 2000), combined Chandra-RXTE (Thompson et al. 2005) and XMMRXTE (Thompson et al. 2008) observations. Though the highenergy data of GS 1826-238 are acceptably fit by a simple exponentially-cutoff power-law model with $E_{\mathrm{c}} \sim 50 \mathrm{keV}$ (e.g. Del Sordo et al. 1999), a less empirical Comptonisation model, coMPTT (Titarchuk 1994), yields a better, physically robust scenario where soft seed photons, whose distribution is approximated by a Wien law, are up-scattered to high energies by a hot $\left(k T_{\mathrm{e}} \sim 20 \mathrm{keV}\right)$ electron plasma. Along with Comptonisation, either a pure blackbody (BB) spectral component (in 't Zand et al. $1999)$ or another low-temperature $(\sim 5 \mathrm{keV})$ Comptonised component (Thompson et al. 2005) are included in the spectral fits.

Recent INTEGRAL results (Cocchi et al. 2010) partly contradict the above results, as only the main, high-energy, Comptonised component was observed. But, as already pointed out by the same authors, the non-detection of the softer component is likely due to the limited passband of JEM-X $(E>$ $3-4 \mathrm{keV})$.

Reflection of Comptonised radiation by an optically thick accretion disk was introduced by Barret et al. (2000) to describe their $R X T E$ data. Detection of a $\sim 6 \mathrm{keV}$ Fe line, never observed by BeppoSAX, was also reported by the same authors. Evidence of another, transient ultrasoft blackbody component, likely related to observed time irregularities in the bursting behaviour, has recently been reported by Thompson et al. (2008).

GS 1826-238 is a peculiar type I X-ray burster. X-ray bursts were found to quasi-periodically recur in a few hours (Ubertini et al. 1999). This is fairly common among bursters, but what makes GS 1826-238 almost unique among its class is the remarkable stability of the recurrence on a timescale of several years (Cocchi et al. 2001). Burst recurrence is clearly related to the persistent emission (Cornelisse et al. 2003), the event frequency increasing with the measured X-ray flux in a limit-cycle fashion (see e.g. Galloway et al. 2004, for a review on the topic).

The lack of evidence for photospheric radius expansion during the bursts (in 't Zand et al. 1999), together with general considerations of the characteristics of the optical counterpart (low mass, short orbital period) constrain the value of the distance to the source in the $\sim 4-9 \mathrm{kpc}$ range (Barret et al. 2000).

In this paper we present wide band spectral analysis of the whole BeppoSAX-NFI record of GS 1826-238. In the next section observations and data analysis methods and details will be given. The applied spectral models and our best-fit results will be presented in Sect. 3, and will be discussed in Sect. 4 in view of the present knowledge of LMXBs spectroscopy.

\section{BeppoSAX observations and data analysis}

The public database of BeppoSAX (Boella et al. 1997a) narrow field instruments (NFI) available at the ASI-ASDC site ${ }^{1}$ includes six pointed NFI observations of GS 1826-238, for a total of $86.7 \mathrm{ks}$ live time for the low-energy concentrator/spectrometer (LECS, Parmar et al. 1995), $175.6 \mathrm{ks}$ for the medium-energy concentrators/spectrometers (MECS, Boella et al. 1997b), $130.9 \mathrm{ks}$ for the high-pressure gas scintillator proportional counter (HPGSPC, Manzo et al. 1995), and $213.8 \mathrm{ks}$ for the Phoswich detection system (PDS, Frontera et al. 1995).

The four NFI instruments (actually, MECS was a set of three concentrators and PDS a set of four detector units) allowed for a wide band energy coverage, nominally in the range $0.1-220 \mathrm{keV}$, with very good overall sensitivity, so the BeppoSAX-NFI capabilities are optimal for an unbiased study of the characteristics of the high-energy, Comptonised emission of NS-LMXBs, along with the physical parameters (temperatures, radii) of both the directly observed and the Compton-modified blackbody (or blackbody-like) seed photon populations.

In Table 1, details of the six analysed BeppoSAX observations are given. We label the observations according to their observing period (OP) number, as already done in the BeppoSAX archive. In this spectroscopic study, only the steady emission of GS 1826-238 was taken into account: time intervals when X-ray bursting events occurred were excluded to avoid possible low-energy spectral contamination from the burst emission. X-ray bursts typically last $\sim 150 \mathrm{~s}$ in the concentrator units, much less in the PDS due to their soft ( $\sim 2 \mathrm{keV}$ blackbody) spectra. A number of bursts were actually detected in some of the pointings (see Table 1). Whenever the (average) burst wait time could be inferred, the period is also reported in the table.

Data analyses of four out of the six BeppoSAX observations have already been reported in literature. Of the measurements performed in 1997, the first three (OPs 1846, 2592, 2699) have been summarised by DelSordo et al. (1999), while OP 2739-41 data were analysed in detail by in 't Zand et al. (1999). Results concerning the last two pointings (OP 7786-7 and 8959-60), respectively performed in 1999 and 2000, were previously unreported. During these last observations GS 1826-238 was more intense than in 1997 and the pointings were longer, thus yielding better counting statistics.

The data were reduced and extracted by the standard SAXDAs ${ }^{2}$ pipeline. To optimise the signal, an extraction radius of $8^{\prime}$ was chosen for the concentrators. A total MECS spectrum was obtained by standard accumulation of the counts in each operating unit. The typical spikes occasionally polluting the PDS count rate $^{3}$ were accurately removed from the good time intervals by setting dedicated thresholds in the lightcurves. For each observing epoch, the corresponding matrices and ancillary response files from the standard BeppoSAX calibration library ${ }^{2}$ were applied. As the LECS response slightly depends on the source position in the detector coordinates, the corresponding corrections were taken into account. The background is automatically subtracted by the pipeline for the instruments with rocking collimators (HPGSPC, PDS), while the available standard background model files were used for the concentrators. The spectra were eventually analysed and best-fitted by the standard software tools of the XSPEC package ${ }^{4}$, version 12.

\footnotetext{
1 http://www.asdc.asi.it

2 http://wwW.asdc.asi.it/bepposax/software/index.html

http://heasarc.gsfc.nasa.gov/docs/sax/abc/saxabc/ saxabc.html

4 http://heasarc.nasa.gov/docs/xanadu/xspec/index.html
} 
Table 1. Observation log.

\begin{tabular}{|c|c|c|c|c|c|c|}
\hline BeppoSAX Obs. Period & OP 1846 & OP 2592 & OP 2699 & OP 2739-41 & OP 7786-87 & OP 8959-60 \\
\hline obs. date & 1997, Apr. 6 & 1997, Oct. 6 & 1997, Oct. 17 & 1997, Oct. 25 & 1999, Oct. 20 & 2000, Apr. 18 \\
\hline LECS live/elapsed time (ks) & $7.2 / 24.6$ & 7.6/46.4 & $9.7 / 70.0$ & $6.8 / 58.3$ & 24.3/156.7 & 31.1/139.4 \\
\hline $\mathrm{MECS}^{a}$ live/elapsed time (ks) & $21.4 / 40.8$ & $21.7 / 46.4$ & $32.3 / 72.1$ & $30.4 / 65.7$ & $30.7 / 161.5$ & 39.1/139.6 \\
\hline HPGSPC exposure (ks) & 10.2 & 9.8 & 14.5 & 13.7 & 45.0 & 37.7 \\
\hline PDS exposure $(\mathrm{ks})$ & 16.5 & 20.3 & 27.9 & 25.6 & 65.5 & 58.0 \\
\hline$\#$ of bursts ${ }^{b}$ & 2 & 1 & 1 & 1 & 4 & 5 \\
\hline average burst wait time (h) & 6.4 & - & - & - & 5.3 & 4.4 \\
\hline References $^{c}$ & (1) & (1) & (1) & (2) & none & none \\
\hline ASM intensity $(\mathrm{mCrab})^{d}$ & $37 \pm 2$ & $26 \pm 3$ & $33 \pm 3$ & $32 \pm 2$ & $40 \pm 5$ & $45 \pm 2$ \\
\hline
\end{tabular}

Notes. ${ }^{(a)}$ MECS units 1,2,3 only for OP 1846; MECS units 2,3 otherwise. ${ }^{(b)}$ All MECS detections except the burst in OP 2592, detected by HPGSPC. ${ }^{(c)}$ Spectroscopic info already available in the literature: (1) Del Sordo et al. (1999); (2) in 't Zand et al. (1999). ${ }^{(d)}$ Average at the epoch of the BeppoSAX observations, 2-10 keV (http://heasarc.gsfc.nasa.gov/docs/xte/asm_products.html).

Systematics likely affect the spectra of the BeppoSAX instruments outside the nominal passbands typically adopted for the fits; these are $0.12-4 \mathrm{keV}, 1.65-10.5 \mathrm{keV}, 7-34 \mathrm{keV}$, and $15-$ $220 \mathrm{keV}$ for LECS, MECS, HPGSPC, and PDS, respectively ${ }^{3}$. We found our spectral points in complete agreement with the above general rule. Even slight extensions of the passbands were found to be possible, e.g. down to $5 \mathrm{keV}$ for HPGSPC and $13 \mathrm{keV}$ for PDS. Conversely, the MECS lower limit had to be set to $2.0 \mathrm{keV}$ in some cases.

Owing to cross calibration uncertainties between the four instruments, whose effects are expected to vary with the observation epoch ${ }^{3}$, a constant normalisation factor was included for each instrument in each fit. The MECS concentrator was kept as a reference, allowing the constants of the other instruments to vary. Standard, typically accepted cross calibration constant values are 0.7-0.9 (LECS), 1.0-1.1 (HPGSPC), and 0.7-0.9 (PDS). The constants we found agree with the above values. A slight, systematic increase with time of the HPGSPC constant is found, likely the effect of a less accurate cross calibration in the late (1999-2000) epochs.

No systematics, accounting for possible instrumental miscalibration, had to be included for both the concentrators and the HPGSPC detector. In the last four observations, the PDS spectra need $2 \%$ systematics, a value commonly adopted to handle intrinsic PDS calibration uncertainties.

\section{Spectroscopic results}

As already mentioned in Sect. 1, most of the energy output in the wide band spectra of NS-LMXBs in LHS generally comes from inverse Comptonisation of a population of soft seed photons by a hot electron plasma cloud. GS 1826-238 is no exception, because whenever non-empirical Comptonisation models were applied to its observations, electron plasma temperatures of $\sim 20 \mathrm{keV}$ were obtained (e.g. in 't Zand et al. 1999; Thompson et al. 2005; Cocchi et al. 2010). Along with Comptonised radiation, a soft component is generally detected in GS 1826-238 spectra, not unlike the other sources of its class (the only exception are the aforementioned recent INTEGRAL results, Cocchi et al. 2010). The modelisation of such a soft component is not straightforward. In an eastern-like scenario, disk blackbody radiation (either multicolour or single temperature) was proposed (Del Sordo et al. 1999, BeppoSAX data, and Barret et al. 2000, RXTE); this agrees more with the classic BeppoSAX picture of NS-LMXB hard states (e.g. Guainazzi et al. 1998). Conversely, a western-like model was proposed by in 't Zand et al. (1999) (hot, $\sim 3 \mathrm{keV}$, NS-originated blackbody). Finally, Comptonised emission by a separate colder electron corona $\left(k T_{\mathrm{e}} \sim 5 \mathrm{keV}\right)$ was invoked by Thompson et al. (2005, RXTE data) and Thompson et al. (2008, Chandra + RXTE data).

\section{Fitting the canonical model}

The standard LHS eastern-like spectral model, i.e. directly observed disk blackbody plus Comptonisation of NS seed photons, was implemented by the XsPEc's formula WABS*(BBODYRAD+COMPTT) and fit to our six observations. The WABS component takes the interstellar hydrogen absorption into account, which is modelled by a Wisconsin absorber (Morrison \& McCammon 1995), while comptт is the widely adopted Comptonisation model by Titarchuk (1994). This model adequately fits the whole data set (see Table 2, bottom). Disk blackbody colour temperatures are constrained in the $0.5-0.6 \mathrm{keV}$ range, and the radii of the (assumed spherical) emitting regions are of the order of $7 \mathrm{~km}$ (a distance to GS 1826-238 of $7 \mathrm{kpc}$ is assumed). The seed photon population of the Comptonised emission, associated to the NS or the inner regions (e.g. a boundary layer, BL) is significantly hotter $(1.2-1.4 \mathrm{keV})$, as expected. The plasma temperatures decrease from $\sim 23 \mathrm{keV}$ in 1997 to $\sim 18 \mathrm{keV}$ in 2000; at the same time, the bolometric fluxes increase by $\sim 20 \%$. This agrees with an expected effect of coronal Compton cooling.

A western-like model with hot (NS) direct blackbody emission and colder (disk) Comptonised seed photons (as the one proposed in in 't Zand 1992) also yields acceptable fits to the data, apart from the case of the 2000 observation (reduced $\chi^{2}$ of $1.27,335$ d.o.f.). But the resulting blackbody parameters (NS colour temperatures well in excess of $3 \mathrm{keV}$ and emitting regions of $\sim 0.1-0.3 \mathrm{~km}$ ) look unlikely, if not unphysical. Also the $N_{\mathrm{H}}$ parameter looks systematically underestimated $(\sim 0.6-$ $\left.0.9 \times 10^{21} \mathrm{~cm}^{-2}\right)$ with respect to the interpolated one $(\sim 1.7 \times$ $10^{21} \mathrm{~cm}^{-2}$ ) obtained by the NH ftool (Dickey \& Lockman 1990).

The presence of a second, colder, Comptonising region was claimed by Thompson et al. (2005). Applying their model to our data also yields acceptable fits, but this, as already discussed in detail in Cocchi et al. (2010) for the case of INTEGRAL data of GS 1826-238, is not unexpected, because this double-Compton model has more free parameters than the spectra request. And, actually, a canonical model with just one Compton corona could describe the spectra in Thompson et al. (2005) just as well (see Table 3 in the cited paper). 
Table 2. Best-fit parameters for the models described in Sect. 3. All errors are given at $90 \%$ confidence level.

\begin{tabular}{|c|c|c|c|c|c|c|}
\hline data set (OP) & 1846 & 2592 & 2699 & $2739-41$ & $7786-87$ & $8959-60$ \\
\hline \multicolumn{7}{|c|}{ WABS $^{*}(\text { СомРTB }+ \text { СомРТВ })^{a}$} \\
\hline$N_{\mathrm{H}}\left(10^{21} \mathrm{~cm}^{-2}\right)$ & $1.14_{-0.24}^{+0.27}$ & $1.24_{-0.26}^{+0.24}$ & $1.03_{-0.20}^{+0.23}$ & $0.82_{-0.22}^{+0.25}$ & $0.86_{-0.13}^{+0.14}$ & $0.86_{-0.12}^{+0.13}$ \\
\hline$N_{\mathrm{H}}(\mathrm{TBABS})\left(10^{21} \mathrm{~cm}^{-2}\right)^{b}$ & $1.55_{-0.30}^{+0.20}$ & $1.66_{-0.35}^{+0.39}$ & $1.38_{-0.27}^{+0.31}$ & $1.11_{-0.31}^{+0.36}$ & $1.15_{-0.17}^{+0.18}$ & $1.15_{-0.15}^{+0.17}$ \\
\hline $\operatorname{disk} k T_{\mathrm{s}}(\mathrm{keV})$ & $0.48 \pm 0.01$ & $0.44 \pm 0.02$ & $0.47 \pm 0.01$ & $0.48 \pm 0.01$ & $0.49 \pm 0.01$ & $0.48 \pm 0.01$ \\
\hline$N S k T_{\mathrm{s}}(\mathrm{keV})$ & $1.92_{-0.18}^{+0.16}$ & $1.49_{-0.18}^{+0.16}$ & $2.02_{-0.17}^{+0.15}$ & $2.20_{-0.16}^{+0.17}$ & $2.15 \pm 0.11$ & $1.68_{-0.10}^{+0.09}$ \\
\hline$k T_{\mathrm{e}}(\mathrm{keV})$ & $24.4_{-2.3}^{+3.1}$ & $21.1_{-1.5}^{+1.8}$ & $20.5_{-1.2}^{+1.6}$ & $20.3_{-1.4}^{+1.6}$ & $21.0_{-1.1}^{+1.2}$ & $19.2 \pm 0.8$ \\
\hline$\alpha$ & $0.98 \pm 0.03$ & $0.94 \pm 0.03$ & $0.95 \pm 0.03$ & $0.95_{-0.04}^{+0.03}$ & $0.95 \pm 0.02$ & $0.98 \pm 0.02$ \\
\hline LECS const. $^{c}$ & $0.847_{-0.013}^{+0.012}$ & $0.811 \pm 0.012$ & $0.827_{-0.012}^{+0.011}$ & $0.850 \pm 0.013$ & $0.827 \pm 0.008$ & $0.877 \pm 0.007$ \\
\hline HPGSPC const. ${ }^{d}$ & $1.045 \pm 0.017$ & $1.042 \pm 0.018$ & $1.040 \pm 0.015$ & $1.010_{-0.015}^{+0.016}$ & $1.087 \pm 0.012$ & $1.149 \pm 0.012$ \\
\hline PDS const. ${ }^{e}$ & $0.935_{-0.029}^{+0.028}$ & $0.957_{-0.028}^{+0.029}$ & $0.895_{-0.026}^{+0.027}$ & $0.855_{-0.026}^{+0.027}$ & $0.890 \pm 0.018$ & $0.993_{-0.021}^{+0.022}$ \\
\hline$\tau^{f}$ & $3.4^{-0.029}$ & 3.9 & $3.9^{-0.020}$ & $3.9^{-0.020}$ & 3.8 & $4.0^{-0.021}$ \\
\hline$R_{\mathrm{bb}}^{f}$ & $14,0.3$ & $14,0.3$ & $15,0.3$ & $14,0.2$ & $14,0.3$ & $15,0.5$ \\
\hline$C A F^{f}$ & $3.8,2.6$ & $3.9,2.8$ & $3.8,2.5$ & $3.8,2.4$ & $3.8,2.5$ & $3.6,2.5$ \\
\hline total flux ${ }^{g}\left(F_{\text {ТОТ }}\right)$ & 1.922 & 1.927 & 1.956 & 1.967 & 2.250 & 2.349 \\
\hline$F(\text { disk })^{h}$ & $1.377(72 \%)$ & $1.384(72 \%)$ & $1.438(74 \%)$ & $1.436(73 \%)$ & $1.631(72 \%)$ & $1.611(69 \%)$ \\
\hline$F(\mathrm{NS})$ & $0.545(28 \%)$ & $0.543(28 \%)$ & $0.518(26 \%)$ & $0.531(27 \%)$ & $0.619(28 \%)$ & $0.738(31 \%)$ \\
\hline Luminosity $^{i}\left(10^{37} \mathrm{erg} \mathrm{s}^{-1}\right)$ & 1.13 & 1.13 & 1.15 & 1.15 & 1.32 & 1.38 \\
\hline red. $\chi^{2}$ (d.o.f.) & $1.035(359)$ & $0.943(352)$ & $1.143(352)$ & $1.093(348)$ & $1.101(350)$ & $1.100(335)$ \\
\hline \multicolumn{7}{|c|}{ WABS $*($ CоMPTT+CoMPTT $)$} \\
\hline$N_{\mathrm{H}}\left(10^{21} \mathrm{~cm}^{-2}\right)$ & $1.11_{-0.24}^{+0.25}$ & $1.14_{-0.26}^{+0.30}$ & $1.00_{-0.20}^{+0.21}$ & $0.86_{-0.22}^{+0.24}$ & $0.84 \pm 0.13$ & $0.75 \pm 0.12$ \\
\hline $\operatorname{disk} k T_{\mathrm{s}}(\mathrm{keV})$ & $0.57 \pm 0.01$ & $0.53 \pm 0.02$ & $0.56 \pm 0.01$ & $0.56 \pm 0.01$ & $0.58 \pm 0.01$ & $0.58 \pm 0.01$ \\
\hline$N S k T_{\mathrm{s}}(\mathrm{keV})$ & [1.8] & $1.54_{-0.16}^{+0.18}$ & [1.8] & [1.8] & [1.8] & $1.82 \pm 0.12$ \\
\hline$k T_{\mathrm{e}}(\mathrm{keV})$ & $24.8_{-2.6}^{+3.8}$ & $22.7_{-2.3}^{+2.10}$ & $19.6_{-1.1}^{+1.3}$ & $18.9_{-1.1}^{+1.2}$ & $19.5_{-0.8}^{+0.9}$ & $19.4_{-0.8}^{+0.9}$ \\
\hline$\tau^{j}$ & $3.83_{-0.44}^{+0.38}$ & $4.09_{-0.30}^{+0.39}$ & $4.70_{-0.26}^{+0.24}$ & $4.88_{-0.26}^{+0.25}$ & $4.79_{-0.18}^{+0.17}$ & $4.47_{-0.20}^{+0.19}$ \\
\hline LECS const. & $0.848 \pm 0.013$ & $0.811 \pm 0.012$ & $0.826_{-0.011}^{+0.012}$ & $0.847 \pm 0.013$ & $0.826 \pm 0.008$ & $0.877 \pm 0.007$ \\
\hline HPGSPC const. & $1.049 \pm 0.016$ & $1.047_{-0.018}^{+0.017}$ & $1.052_{-0.014}^{+0.011}$ & $1.025 \pm 0.014$ & $1.102 \pm 0.011$ & $1.153 \pm 0.012$ \\
\hline PDS const. & $0.946_{-0.025}^{+0.024}$ & $0.964_{-0.029}^{+0.025}$ & $0.914_{-0.025}^{+0.026}$ & $0.882 \pm 0.026$ & $0.910 \pm 0.018$ & $0.997 \pm 0.021$ \\
\hline$F_{\mathrm{TOT}}$ & 1.892 & 1.925 & 1.922 & 1.917 & 2.204 & 2.336 \\
\hline$F($ disk $)$ & $1.497(79 \%)$ & $1.443(75 \%)$ & $1.582(82 \%)$ & $1.603(84 \%)$ & $1.822(83 \%)$ & $1.714(73 \%)$ \\
\hline$F(\mathrm{NS})$ & $0.395(21 \%)$ & $0.482(25 \%)$ & $0.340(18 \%)$ & $0.314(16 \%)$ & $0.382(17 \%)$ & $0.622(27 \%)$ \\
\hline red. $\chi^{2}$ (d.o.f.) & $1.042(360)$ & $0.933(352)$ & $1.166(353)$ & $1.121(349)$ & $1.166(351)$ & $1.106(335)$ \\
\hline \multicolumn{7}{|c|}{ WABS $*($ BB + CoMPTT $)$} \\
\hline$N_{\mathrm{H}}\left(10^{21} \mathrm{~cm}^{-2}\right)$ & $1.59_{-0.23}^{+0.25}$ & $1.64_{-0.23}^{+0.25}$ & $1.51_{-0.19}^{+0.24}$ & $1.44_{-0.23}^{+0.25}$ & $1.36 \pm 0.13$ & $1.35 \pm 0.1$ \\
\hline$R_{\mathrm{bb}}(\mathrm{km})^{k}$ & $6.8 \pm 0.2$ & $7.8 \pm 0.3$ & $7.2_{-0.2}^{-0.19}$ & $7.2 \pm 0.3$ & $7.0 \pm 0.2$ & $7.7 \pm 0.2$ \\
\hline $\operatorname{disk} k T_{\mathrm{bb}}(\mathrm{keV})$ & $0.57 \pm 0.01$ & $0.53 \pm 0.02$ & $0.56 \pm 0.01$ & $0.56 \pm 0.01$ & $0.58 \pm 0.01$ & $0.58 \pm 0.01$ \\
\hline$N S k T_{\mathrm{s}}(\mathrm{keV})$ & $1.40 \pm 0.08$ & $1.25 \pm 0.07$ & $1.32_{-0.09}^{+0.07}$ & $1.28_{-0.09}^{+0.08}$ & $1.41 \pm 0.07$ & $1.37 \pm 0.05$ \\
\hline$k T_{\mathrm{e}}(\mathrm{keV})$ & $23.5_{-2.3}^{+3.2}$ & $21.5_{-1.7}^{+2.1}$ & $18.8 \pm 1.1$ & $18.0_{-1.0}^{+1.1 .1}$ & $18.8_{-0.7}^{+0.8}$ & $18.4_{-0.6}^{+0.7}$ \\
\hline$\tau^{j}$ & $4.04_{-0.43}^{+0.36}$ & $4.31_{-0.33}^{+0.31}$ & $4.94_{-0.25}^{+0.26}$ & $5.15_{-0.26}^{+0.0}$ & $\begin{array}{l}4.08_{-0.18}^{+0.7} \\
4.17\end{array}$ & $4.76_{-0.17}^{+0.16}$ \\
\hline LECS const. & $0.848 \pm 0.012$ & $0.812 \pm 0.012$ & $0.827_{-0.012}^{+0.011}$ & $0.847 \pm 0.013$ & $0.827 \pm 0.008$ & $0.877 \pm 0.007$ \\
\hline HPGSPC const. & $1.061_{-0.016}^{+0.017}$ & $1.053 \pm 0.017$ & $1.057 \pm 0.015$ & $1.031 \pm 0.015$ & $1.102 \pm 0.012$ & $1.162 \pm 0.011$ \\
\hline PDS const. & $0.974 \pm 0.027$ & $0.975 \pm 0.027$ & $0.920_{-0.027}^{0.026}$ & $0.887_{-0.026}^{0.027}$ & $0.912 \pm 0.018$ & $1.000_{-0.021}^{0.022}$ \\
\hline$F_{\text {TOT }}$ & 1.879 & 1.915 & 1.916 & 1.914 & 2.206 & 2.331 \\
\hline$F$ (сомPTт disk) & $1.636(87 \%)$ & $1.696(88 \%)$ & $1.681(88 \%)$ & $1.688(88 \%)$ & $1.929(87 \%)$ & $2.043(88 \%)$ \\
\hline$F$ (NS blackbody) & $0.243(13 \%)$ & $0.219(12 \%)$ & $0.235(12 \%)$ & $0.226(12 \%)$ & $0.277(13 \%)$ & $0.288(12 \%)$ \\
\hline red. $\chi^{2}$ (d.o.f.) & $1.084(359)$ & $0.945(352)$ & $1.223(352)$ & $1.163(348)$ & $1.242(350)$ & $1.181(335)$ \\
\hline
\end{tabular}

Notes. ${ }^{(a)}$ The values of the сомртв parameters $\delta, \log A$ and $\gamma$ are kept frozen to 0,8 and 4 , respectively (see Sect. 3). ${ }^{(b)} N_{\mathrm{H}}$ value determined by the TBABS absorber (see Sect. 3). ${ }^{(c)}$ LECS/MECS normalisation ratio (see Sect. 2). ${ }^{(d)}$ HPGSPC/MECS normalisation ratio. ${ }^{(e)}$ PDS/MECS normalisation ratio. ${ }^{(f)}$ Derived parameter (see Sect. 3). Spherical geometry assumed for $\tau$ calculation; CAFs and $R_{\mathrm{bb}}$ are given for the disk and the NS populations, respectively. ${ }^{\left({ }^{)}\right)}$All fluxes in $10^{-9} \mathrm{erg} \mathrm{cm}^{-2} \mathrm{~s}^{-1}, 0.1-200 \mathrm{keV}$, unabsorbed (the absorbed flux is always $\lesssim 1 \%$ of $F_{\text {TOT }}$ ). ${ }^{(h)}$ Percentage with respect to $F_{\text {TOT }}$ is also given. ${ }^{(i)}$ Unabsorbed, $0.1-200 \mathrm{keV}$, assumed distance of $7 \mathrm{kpc} .{ }^{(j)}$ Assumed spherical geometry. ${ }^{(k)}$ For an assumed distance of $7 \mathrm{kpc}$.

\section{A two-seed Comptonisation model}

These BeppoSAX data clearly indicate that there are two distinct soft photon populations in GS 1826-238. Assuming just one population (by imposing the same value to the $k T_{B B}$ and the $k T_{\text {s }}$ parameters in the model above) leads to unacceptable best fits. Nevertheless, whether the disk blackbody is totally unaffected by Comptonisation is a questionable hypothesis. Actually, a model that assumes both the populations (NS-BL and disk) to be Compton-modified by the plasma corona fits the whole data 
set with even slightly better $\chi^{2}$ statistics. The best-fit parameters of this model, implemented as WABS*(сомPTT+COMPTT), are displayed in Table 2. Here we assume the electron cloud parameters of the two comptT components $\left(k T_{\mathrm{e}}\right.$ and $\left.\tau\right)$ to be identical, similar to what was already done in Titarchuk \& Shaposhnikov (2005). In that paper, the spectral evolution of the NS LMXB $4 \mathrm{U} 1728-34$ was described by a BMC+BMc model with tied $\alpha$ indexes and different seed photon temperatures.

The physical parameters of the best fits are in good agreement with those of the classic model discussed above. The NS-BL population temperature is not well constrained by this model in four observations, with best-fit values in excess of $2 \mathrm{keV}(\sim 2.2-2.9 \mathrm{keV})$ but also with the fit not very sensitive to lower, more likely, $k T_{\mathrm{s}}$ values. In these cases, we therefore froze the NS-BL temperature to $1.8 \mathrm{keV}$. It is important to stress that this two-seed Comptonisation model has the same number of free parameters as the canonical one, thus keeping the problem's degrees of freedom constant. This is an intrinsically different model with respect to the one proposed in Thompson et al. (2005) and Thompson et al. (2008), in which two separate Compton plasmas with different temperatures, optical depths, and geometries are hypothesised.

\section{Applying the СОМРТв model}

The use of сомтт,, which assumes Wien-distributed seed photons, could explain the above difficulties in constraining the NS-BL population temperature. The recently developed Comptonisation model by Farinelli et al. (2008, сомРтв) is a valid tool to deal with Planck-distributed or even pseudoblackbody distributed seed photons. In fact, in сомРтв the form of the seed photons energy distribution is given by

$B B(x) \propto \frac{x^{\gamma}}{\mathrm{e}^{x}-1}$,

where $x \equiv E / k T_{B B}$ is the adimensional energy, and $\gamma$ is an index empirically modelling non-ideal blackbody distributions. For $\gamma=3$, which is сомРтв's default, Eq. (1) reduces to a pure Planckian. We therefore replaced сомРтT with сомРтв in our double-seed Compton model. Actually, сомРтв is a more general model including, in a self-consistent way, the seed photons emission along with the Comptonised one, the latter being either purely thermal (as in сомРTT) or mixed thermal plus dynamical (bulk Comptonisation). This is characterised by the model's free parameters $\log A$ and $\delta$ (Farinelli et al. 2008). But in this work we use сомРтв in a purely thermal, Compton-dominated way, which makes it substantially equivalent to сомPTT (apart from the assumption on the distribution of the soft seed photons, which is Wien in сомРтT). In particular, the data do not require direct blackbody emission (i.e. we can assume complete coverage of the two seed regions), and no dynamical effects are to be expected. This means, in practice, to freeze the model parameters $\log A=8$ and $\delta=0$, respectively. This restricted use of COMPTB, which is ideal for NS-LMXBs in LHS, is detailed in Cocchi et al. (2010).

The results of our WABS*(СОмРТВ+СОМРтв) fits are also reported in Table 2 (top). The six best-fit spectra of GS 1826-238 are displayed in Fig. 1, while in Fig. 2 the residuals to the best fits are shown. In Fig. 3 a model comparison with respect to the classic one is also displayed.

One of the main outcomes of the use of сомттв is the evidence of non-ideal distribution of the seed photon populations. In fact, freezing the $\gamma$ index to 3 (pure BB) does not yeld acceptable best fits. On the other hand, when $\gamma$ is a free parameter, the fit is rather insensitive to its value $(\gamma \sim 3.5-4.5$ are acceptable), though it tends to cluster around 3.8. We thus decided to freeze the index of both the seed populations to $\gamma=4$. This value yields a pseudo-blackbody distribution that behaves like a Wien law at the lowest energies, making this assumption consistent with what is obtained using the coMPTT model. Moreover, the number of free parameters of the whole model is still the same as for the classic one.

As expected, the main сомРтв parameter values are in good agreement with the cOMPTT ones. The $\chi^{2}$ statistics we obtain are systematically (even though slightly) better than in the other models. Besides that, in this way the NS-BL population temperature is well constrained in all the observations, with values of $k T_{\mathrm{s}}$ in the range 1.5-2.2, which are physically acceptable for NS colour temperatures (Ebisuzaki 1986). The disk photon population has $k T_{\mathrm{s}} \sim 0.4-0.5$, while the decreasing trend in the plasma $k T_{\mathrm{e}}$ temperatures is confirmed. The disk population temperature is remarkably stable troughout the observations, while the NS-BL one oscillates significantly. With сомРтв, the disk temperature is found to be systematically lower with respect to the one obtained by the traditional вв+сомртт; conversely, the NS-BL temperatures are systematically higher.

Most of the energy output $(>70 \%)$ comes from the Comptonised disk radiation. The relative weight of the two Comptonised components is constant with time, though a stronger NS contribution is observed in 2000 in good correlation with the increased source intensity. A more variable ratio is obtained by the two-seed compTT model, but this is likely a bias from the freezing of the NS $k T_{\mathrm{s}}$ in four observations. (Not surprisingly, they are the ones with the lowest resulting NS contributions.)

The energy index of the Compton Green function, $\alpha$, is well constrained in the $0.94-0.98$ range. An $\alpha$ value of $\sim 1$ is typically observed in NS-LMXB systems (e.g. Cocchi et al. 2010 for the case of GS 1826-238), and the theoretical reasons for this peculiarity have recently been addressed in Farinelli \& Titarchuk (2011).

Interestingly, the best-fit column absorption $N_{\mathrm{H}}$ values as obtained by the WABs absorber are slightly, but systematically, lower than the predicted one (Dickey \& Lockman 1990, see above). This is more evident for the double-seed Comptonised models than for the canonical eastern-like one and could testify a limitation of this kind of model. On the other hand, the $\mathrm{NH}$ ftool, based on a table interpolation, could overestimate the absorption in the particular case of the GS 1826-238 direction. Actually, the WABs component, based on rather outdated relative abudances, was adopted in order to directly compare our results with the ones already reported in BeppoSAX literature. Indeed, the application of the more up-to-date Tuebingen-Boulder absorption model (твABs) and corresponding abundances (Wilms et al. 2000) to our data leads to higher, more likely, $N_{\mathrm{H}}$ values. Such values are also reported in Table 2.

сомртв best fits allow us to derive additional interesting information about some physical parameters. For example, the plasma cloud optical depths $\tau$, derived by the Eqs. (17)-(24) in Titarchuk \& Lyubarskij (1995) with assumed spherical geometry, are included in Table 2. The amount of the energy amplification due to the Compton scattering, expressed by the Compton amplification factor $(C A F)$, is also tabulated for both the seed populations. Approximated values for the radii of the (assumed spherical) blackbody seed photon regions were also calculated as in Cocchi et al. (2010): radii of $\sim 0.2-0.5 \mathrm{~km}$ and $\sim 13-$ $15 \mathrm{~km}$ were obtained for the NS-BL and disk seed populations, respectively (distance of $7 \mathrm{kpc}$ ). A non-ideal geometry of the 


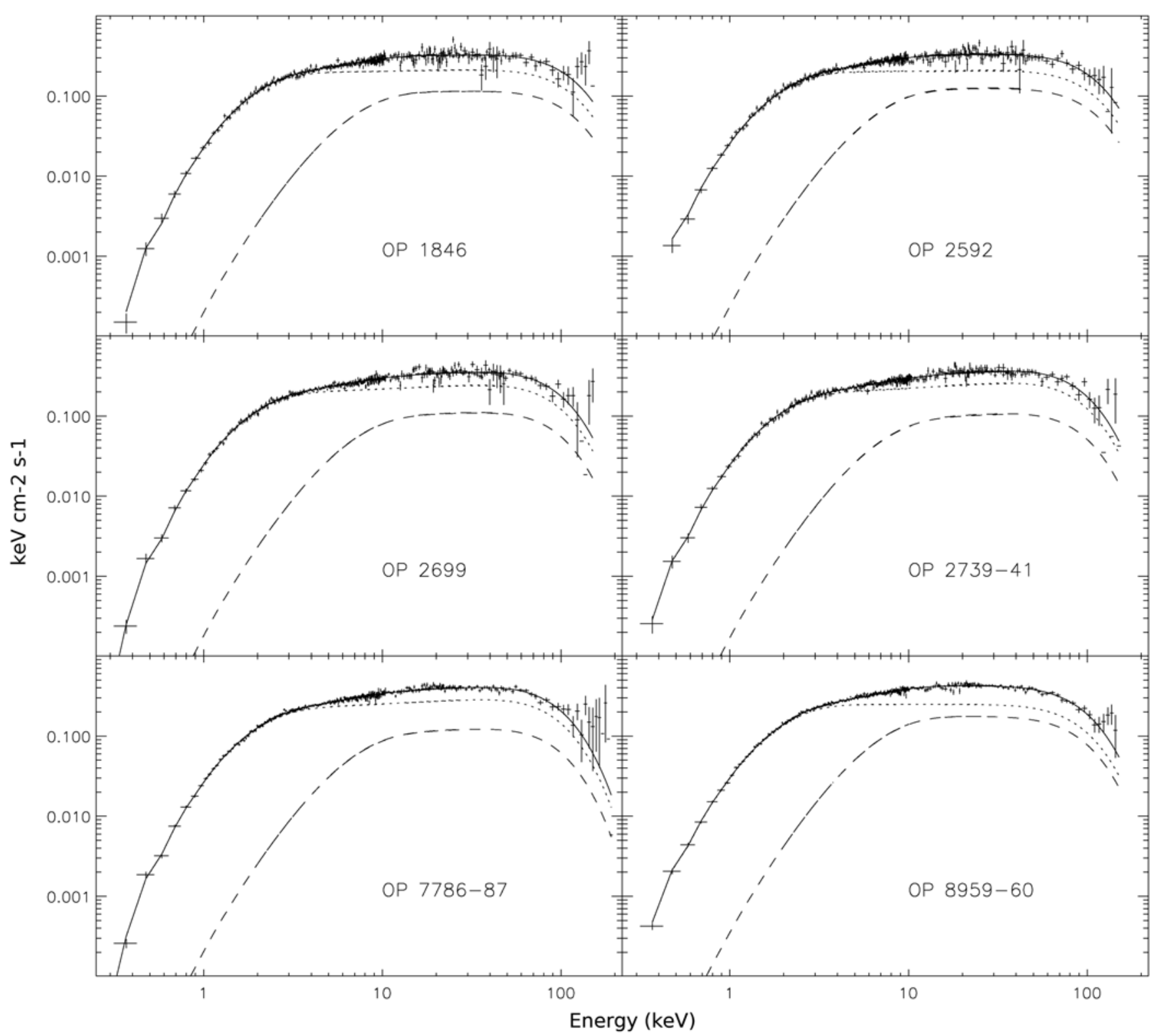

Fig. 1. The six absorption-corrected BeppoSAX-NFI spectra of GS 1826-238. The two Comptonised photon populations of the best fit model (see Table 2, top, and Sect. 3.3) are also plotted: disk seed photons (dots) and NS-BL seed photons (dashes).

NS-BL emitting region (a belt or a hot spot) could explain its relatively small order of magnitude $(<1 \mathrm{~km}$, while NS radii are of the order of $10 \mathrm{~km})$.

\section{Discussion}

Within the context of the broadband LHS emission of NSLMXB, the main results of the present work are: a) the confirmation of the presence of two populations of soft X-ray photons; $b$ ) the possibility that both the populations are (almost) completely Comptonised by an extended electron corona; c) the evidence of non-ideal energy distribution of the seed populations. These items will be discussed below.

\section{Two photon populations}

Our results confirm that two populations of soft photons (at least one Comptonised) characterise the spectroscopic properties of
GS 1826-238. The brighter population is the colder $(\sim 0.5 \mathrm{keV})$ one and can be associated to the emission of an accretion disk. The other, hotter $(\sim 1.5-2.0 \mathrm{keV})$ component, most likely Comptonised by a $\sim 20 \mathrm{keV}$ electron corona, should originate in the inner regions (a boundary/transition layer in the very inner disk or the photosphere of the NS itself) of the X-ray source. Both a directly observed disk emission combined with Comptonised NS radiation and a double-seed (disk and NS/BL) Comptonised emission are effective ways to describe the wide band spectroscopic emission of this source. In this respect GS 1826-238 does not look different from a number of sources belonging to the same class. Actually, according to Cocchi (2011), a general picture is emerging from the BeppoSAX data archive where NS in an LHS can be divided into two main subclasses: two-photon population (2P) and single-photon population (1P) sources. The $2 \mathrm{P}$ sources look intrinsically brighter than the $1 \mathrm{P}$ ones, suggesting the accretion rate $\dot{m}$ (and not, e.g., intrinsic evolutionary properties of the X-ray binary) drives their main spectral differences. In the above picture, GS 1826-238 can be 


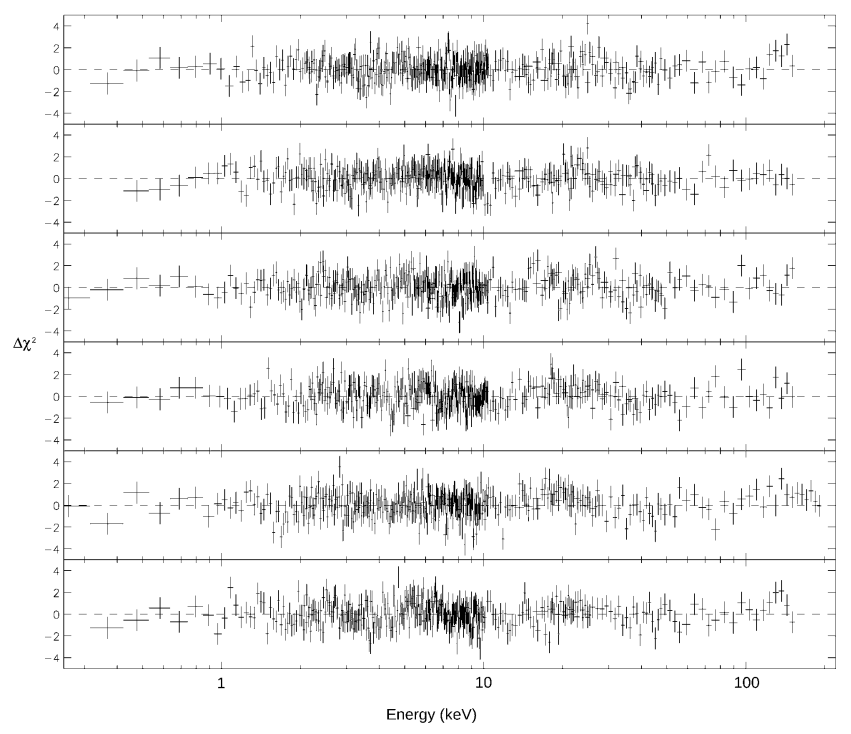

Fig. 2. Fit residuals, in $\chi^{2}$ units, for the two-seed CompTB best fits (chronologically from top to bottom panels, OP 1846 to OP 8959-60).

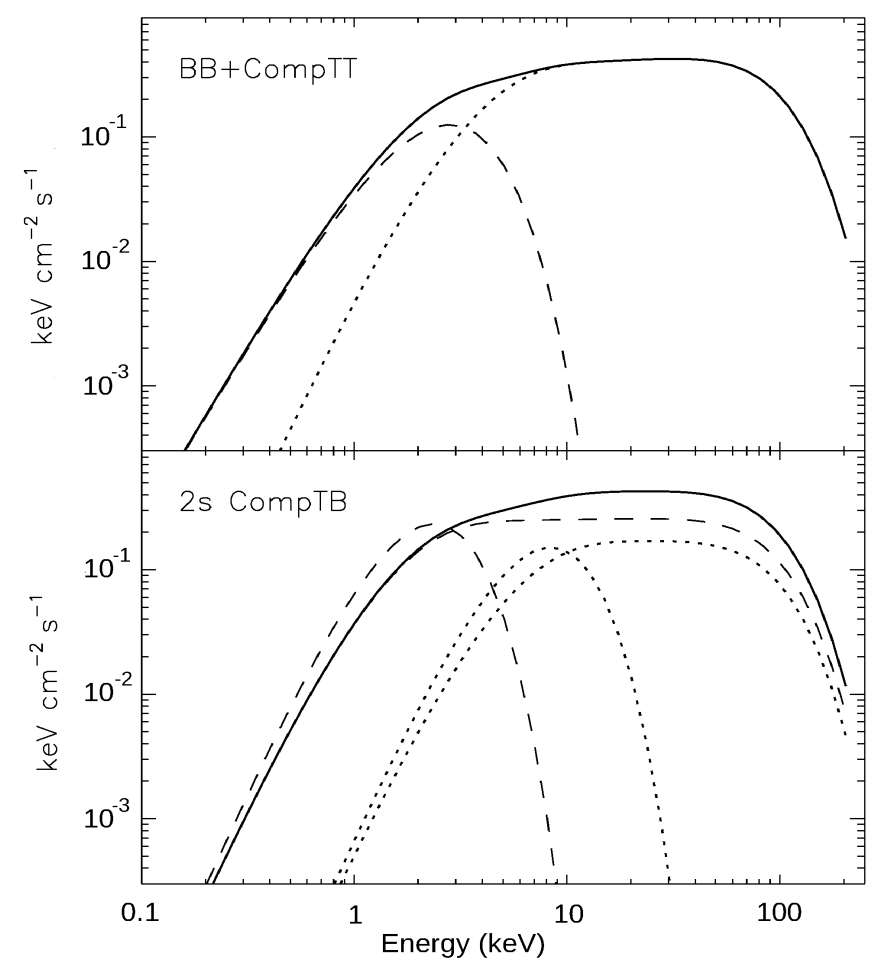

Fig. 3. An example (OP 8959-60) of our double-seed сомртв model (lower panel) compared to a typical вв+сомРтт model (upper). Along with the two Comptonised components (disk and NS-BL, dashses and dots, respectively), the original pseudo-blackbody distribution of the two components is plotted with the same linestyle.

regarded as a clear example of a 2P LHS source. Its luminosity, in excess of $10^{37} \mathrm{erg} \mathrm{s}^{-1}$, is in good agreement with the above scenario.

\section{Double-seed models and extended coronas}

From the present data analysis, a double-seed Comptonised emission seems a more effective way to describe the wide band emission of GS 1826-238. Again, this agrees with the results of Cocchi (2011) obtained for a number of $2 \mathrm{P}$ sources. A completely (or, more likely, almost-completely) Comptonised disk blackbody-like emission requires a hot plasma corona radially extended enough to geometrically shield the disk. Extended Compton regions are not unexpected in an LHS, where sources are generally believed to accrete in a relatively low $\dot{m}$ regime in which the coronal Compton cooling is far from being efficient as in the case of NS high/soft states.

According to Paizis et al. (2006) and Mainardi et al. (2010), the relative importance of thermal versus dynamical (bulk) Comptonisation is a very effective parameter in modelling the variety of NS-LMXBs spectral states and the state transitions in individual sources. In their scenario, LHS are characterised by a large height scale and radially extended hot electron plasma able to screen, to a distant observer, the bulk Compton amplification effects (occurring very close to the NS). With increasing $\dot{m}$, a state transition to an intermediate state is expected. In this brighter state, an efficient Compton cooling should significantly reduce the height scale of the electron corona, allowing for the direct observation of the bulk-Comptonised NS (and/or inner accretion disk) blackbody radiation that originates the so-called hard tail. At the same time, the disk seed photons, accounting for most of the energy output, are still Comptonised by the electron plasma. Such a description of the intermediate state recalls, in its main features, the characteristics of a western-like model. Therefore a double-seed Comptonised emission (like the one we propose for GS 1826-238) is easier to reconcile with the above framework than the classic eastern-like model, as it implies a more radially extended corona. In fact, this picture of a (relatively luminous) LHS smoothly evolves, with increasing $\dot{m}$ and reduced coronal height scale, into the intermediate state picture of Paizis et al. (2006) and Mainardi et al. (2010).

As already reported in Table 2, in our double-seed Compton (сомртв) model, the disk Comptonised emission dominates the overall energy budget of GS 1826-238, accounting for $\sim 70 \%$ of the total. But this does not mean at all that the accretion disk produces most of the energy output. As the disk blackbody is Compton-amplified by a factor of $\sim 3.8$, one can easily verify that the disk seed photon population accounts for $<20 \%$ of the total energy budget. Most of the output $(\sim 70 \%)$ is due to the Compton boosting and only $\sim 10 \%$ to the NS-BL seed population. This implies that the source central engine (which the plasma cloud belongs to) produces most of the X-ray energy of GS 1826-238, as naturally expected. The total energy of the disk population is greater (by a factor of $\sim 2$ ) than that of the NS-BL one. This difference is even wider in terms of number of photons, as the disk colour temperature is lower. The order of magnitude of the (assumed spherical) corresponding emitting regions (see previous section and Table 2) confirm the disk nature of the cold photon population and the interior origin, NS and/or BL, of the hot photons.

Time analysis, more than spectroscopy, could possibly address the question about the nature (single or double seeded) of the Comptonised emission of GS 1826-238 and similar NSLMXBs. According to Titarchuk \& Shaposhnikov (2007), the presence of two bounded media of different sizes (namely an extended accretion disk and a more compact Compton cloud or BL region) is presumed to yield emerging power density spectra with two characteristic break frequencies, $v_{\mathrm{b}}^{\text {disk }}$ (lower) and $v_{\mathrm{b}}^{\mathrm{BL}}$ (higher). For photon energies close to the peak of the disk seed population $(E \sim 1-2 \mathrm{keV}$ ), a complete Comptonisation (i.e. an extended corona) could strongly reduce the disk signatures in the power spectra, because seed photons are scattered to higher 
energies. Thus, weak or no detection of $v_{\mathrm{b}}^{\text {disk }}$ in a relatively narrow soft X-ray band could support the proposed double-seed Comptonisation model. We plan to investigate this possibility in a forthcoming paper.

\section{Energy and spatial distributions of the seed populations}

With the statistical quality of the BeppoSAX data, it is impossible to disentangle the actual geometry and temperature distributions of the seed photon populations. For example, the disk population is likely multicolour-distributed, but pseudo-blackbody (or, in several cases, even pure blackbody, see Cocchi 2011) suffices for fitting the observed spectra. Moreover, it is not clear whether this double-population scenario reflects the actual presence of two distinct, well-separated photon populations; in fact, the fits could mimic by two blackbody-like populations what is actually a strong and spatially wide temperature gradient between the outer and the inner (close to NS) disk (see also Cocchi 2011).

The first evidence, in our data, of non-Planckian distribution of the seed photon populations is an interesting subject. This result was obtained thanks to the excellent quality of the BeppoSAX observations of GS 1826-238 and to the features of the recently developed сомттв model. But, actually, it is impossible to discern whether this non-ideal distribution is a characteristic of both or just one population (more likely the disk one, because it is brighter and more spacially extended). Moreover, an index $\gamma \sim 4$ could actually mimic an intrinsically multicolour disk emission whose properties are only approximated by Eq. (1). Modified and/or multicolour blackbodies are likely to be observed from X-ray binaries, and the available models are only ideal or empirical approximations: so the blackbody parameters obtained, either colour temperatures (e.g. Ebisuzaki 1986) or radii, should be always regarded as just reasonable suggestions.

Acknowledgements. The data analysis and the initial developments of this work have been supported by the grant from the INAF PRIN 2007 Bulk motion Comptonization models in X-ray Binaries: from phenomenology to physics, P.I. M. Cocchi.
The authors thank L. Titarchuk for fruitful science interaction in the early phase of this work; M. Orlandini for BeppoSAX archive data retrieval and PDS data analysis suggestions and tips; and C. Ferrigno for help in updated HPGSPC software implementation.

\section{References}

Barret, D., Motch, C., \& Pietsch, W. 1995, A\&A, 305, 526

Barret, D., Olive, J. F., Boirin, L., et al. 2000, ApJ, 533, 329

Boella, G., Butler, R. C., Perola, G. C., et al. 1997a, A\&AS, 122, 299

Boella, G., Chiappetti, L., Conti, G., et al. 1997b, A\&AS, 122, 327

Cocchi, M. 2010, AIP Proc., Cesme [arXiv: 1103.1757]

Cocchi, M., Bazzano, A., Natalucci, L., et al. 2001, Adv. Space Res., 28, 375

Cocchi, M., Farinelli, R., Paizis, A., \& Titarchuk, L. 2010, A\&A, 509, A2

Cornelisse, R., in 't Zand, J. J. M., Verbunt, F., et al. 2003, A\&A, 405, 1033

Del Sordo, S., Frontera, F., Pian, E., et al. 1999, Astrophys. Lett. Comm., 38, 125

Dickey, J., \& Lockman, F. 1990, ARA\&A, 28, 215

Ebisuzaki, T., \& Nomoto, K. 1986, ApJ, 305, 67

Farinelli, R., \& Titarchuk, L. 2011, A\&A, 525, A102

Farinelli, R., Titarchuk, L., Paizis, A., \& Frontera, F. 2008, ApJ, 680, 602

Frontera, F., Costa, E., Dal Fiume, D., et al. 1997, A\&AS, 122, 357

Galloway, D. K., Cummings, A., Kuulkers, E., et al. 2004, ApJ, 601, 466

Guainazzi, M., Parmar, A. N., Segreto, A., et al. 1998, A\&A, 339, 802

Mainardi, L., Paizis, A., Farinelli, R., et al. 2010, A\&A, 512, 57

Makino, F. 1988, IAU Circ., 4653

Manzo, G., Giarrusso, S., Santangelo, A., et al. 1997, A\&AS, 122, 341

Mitsuda, K., Inoue, H., Nakamura, N., \& Tanaka, Y. 1989, PASJ, 41, 97

Morrison, R., \& McCammon, D. 1983, ApJ, 270, 119

Paizis, A., Farinelli, R., Titarchuk, L., et al. 2006, A\&A, 459, 187

Parmar, A. N., Martin, D. D. E., Bavdaz, M., et al. 1997, A\&AS, 122, 309

Strickman, M., Skibo, J., Purcell, W., Barret, D., \& Motch, C. 1996, A\&AS, 120, 217

Thompson, T. W. J., Rothschild, R. E., Tomsick, J. A., \& Marshall, H. L. 2005, ApJ, 634, 1261

Thompson, T. W. J., Galloway, D. K., Rothschild, R. E., \& Homer, L. 2008, ApJ, 681,506

Titarchuk, L. 1994, ApJ, 434, 570

Titarchuk, L., \& Lyubarskij, Y. 1995, ApJ, 450, 876

Titarchuk, L., \& Shaposhnikov, N. 2005, ApJ, 626, 298

Titarchuk, L., Shaposhnikov, N., \& Arefiev, V. 2007, ApJ, 660, 556

Ubertini, P., Bazzano, A., Cocchi, M., et al. 1999, ApJ, 514, L27

White, N. E., Stella, L., \& Parmar, A. N. 1988, ApJ, 324, 363

Wilms, J., Allen, A., \& McCray, R. 2000, ApJ, 542, 914

in 't Zand, J. J. M. 1992, Ph.D. Thesis, University of Utrecht

in 't Zand, J. J. M., Heise, J., Kuulkers, E., et al. 1999, A\&A, 347, 891 